\title{
Clostridium difficile colitis - a serious current problem
}

\author{
Irina Magdalena Dumitru ${ }^{1,2^{*}}$, Eugen Dumitru', Roxana Carmen Cernat ${ }^{2}$, Andra Elena Petcu $^{2}$, Carmen llie Șerban², \\ Sorin Rugină ${ }^{1,2}$ \\ From The 9th Edition of the Scientific Days of the National Institute for Infectious Diseases Prof Dr Matei Bals \\ Bucharest, Romania. 23-25 October 2013
}

\section{Background}

Clostridium difficile is currently considered a significant cause of nosocomial infection and is associated with increasing morbidity and mortality. The probability of colonization of hospitalized patients increases with the length of their hospital stay and depends on the local epidemiologic situation.

The study aimed to evaluate the epidemiological, clinical and treatment features of Clostridium difficile colitis (CDC), and the relapse associated risk factors in the Infectious Diseases Clinic and Gastroenterology Clinic.

\section{Methods}

Prospective study of cases admitted to our clinic with $\mathrm{CDC}$ in the last year. The following parameters were analyzed: age, sex, comorbidities, recent hospitalizations, recent surgery, use of antibiotics, use of proton pump inhibitors (PPIs), clinical form of the disease, methods of diagnosis and therapeutic response.

\section{Results}

In the two Clinics, 42 patients were diagnosed and treated in the last year (14 times more than during 2011-2012). More than half of the cases had severe clinical forms (pancolitis). We recorded no case of toxic megacolon. All patients reported use of antibiotics, most frequently fluoroquinolones and cephalosporins (48\% and $34 \%$ ). $68 \%$ of patients had history of prolonged hospitalizations in orthopedics, surgery, oncology or hematology clinics and $82 \%$ of patients had comorbidities (leukemia, cancer, liver cirrhosis). The toxin (A and B) test was positive in only half of cases, in all cases the diagnosis was confirmed by sigmoidoscopy. Metronidazole, vancomycin and rifaximin were administered in $68 \%$ of cases, intravenous metronidazole, ertapenem and rifaximin in $10 \%$ of cases, metronidazole orally and rifaximin in $10 \%$ of cases and only $12 \%$ of cases responded favorably to metronidazole alone. We recorded relapses in 9 patients (21.4\%). Relapse associated risk factors were: malignant diseases, inflammatory bowel disease, IPP treatment, colonic resection, immunosuppressive therapy and absence of rifaximin regimen.

\section{Conclusion}

Interdisciplinary collaboration is vital for limiting the development of this very serious, often fatal disease. Rational use of antibiotic therapy is essential, particularly in an environment contaminated by spores of Clostridium difficile. We need to examine the possibilities of using vaccines to combat infection due to Clostridium difficile in real life and clinical trials.

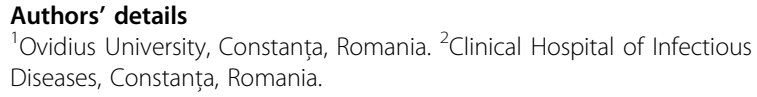

Published: 16 December 2013

doi:10.1186/1471-2334-13-S1-O20

Cite this article as: Dumitru et al:: Clostridium difficile colitis - a serious current problem. BMC Infectious Diseases 2013 13(Suppl 1):O20.

* Correspondence: dumitrui@hotmail.com

${ }^{1}$ Ovidius University, Constanța, Romania

Full list of author information is available at the end of the article 